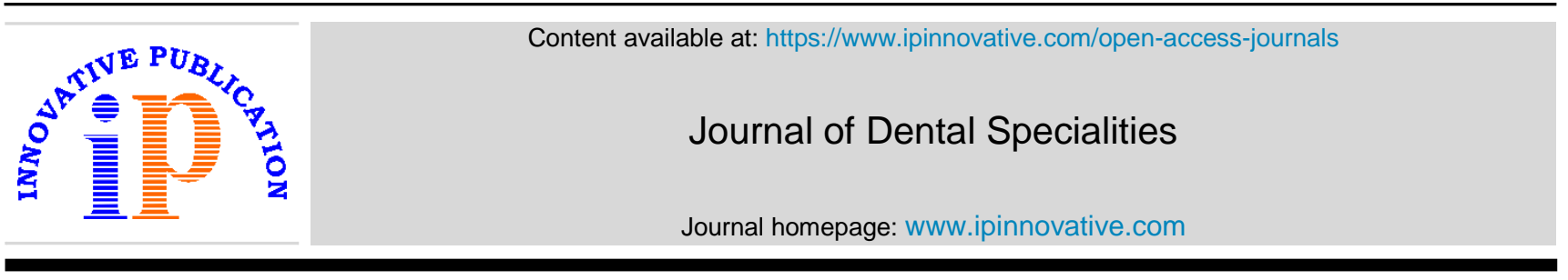

\title{
COVID-19: Challenges, opportunities, and way ahead for dentistry
}

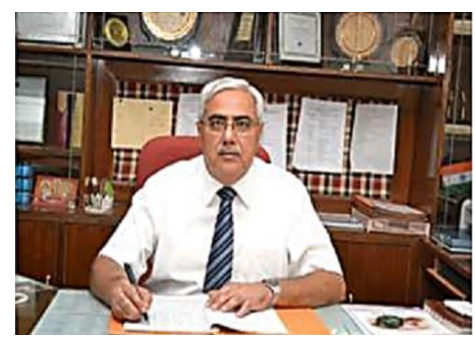

Prof. (Dr.) Vinod Sachdev

Director-PG Studies, I.T.S Centre for Dental Studies and Research, Muradnagar, Uttar Pradesh, India Editor-in-chief

\section{A R T I C L E I N F O}

Article history:

Received 10-10-2020

Accepted 27-10-2020

Available online 23-11-2020

\begin{abstract}
(C) This is an open access article distributed under the terms of the Creative Commons Attribution License (https://creativecommons.org/licenses/by/4.0/) which permits unrestricted use, distribution, and
\end{abstract} reproduction in any medium, provided the original author and source are credited.
COVID-19 has changed the world forever. Every aspect of our life has been impacted from how we live, interact with each other, the way we work, communicate and travel. Decisions made now will have a profound effect on many generations to come and will reshape our society in many lasting ways. People wearing face mask and social distancing have become the new norm. The effects of global health crisis and strategies implemented to ameliorate the pandemic across the globe have had unparalleled consequences on dentistry. Regular dental practice was brought to a virtual standstill during the nationwide imposed lockdown. COVID-19 has seen cessation of all non-urgent dental care in India.

The Ministry of Health and Family Welfare, India, have categorised dentists, auxiliaries as well as patients undergoing dental procedures at high risk of cross-infection as most dental procedures require close contact with the patient's oral cavity, saliva, blood, and respiratory tract secretions. Saliva is rich in COVID 19 viral load. Many patients who are asymptomatic may be carriers. For this reason, it is suggested that all patients visiting a dental office must be treated with due precautions. But we have to understand the fact that we cannot rely only on providing emergency dental care to the patients. A country like India which has large unmet dental needs cannot afford to neglect oral health care delivery as there is shortage of resources to tackle the existing burden of oral diseases. Also delayed dental care can have varied consequences whether it is due to non-provision of regular dental care or reluctance of patient to seek oral health care during the pandemic. Untreated dental caries due to delayed dental care may lead to formation of pus, ulceration, fistula, abscess or other infections eventually increasing the sufferings of patient, thus escalating the cost of treatment and also resulting in poor oral health related quality of life. Routine dental care also provides an opportunity for preventive care, screening for systemic diseases through their oral manifestations which might otherwise be missed. Thus, the recent state of affairs has put forth various challenges and opportunities in re-configuring the dental practice globally.

The key points in reforming and re-structuring dental practice

1. The pandemic obligates the need to strike a balance between the safety of the healthcare professionals yet providing optimum dental care to the patients. Dental health care personnel need to understand the potential risk and implications of transmission of (SARS)- CoV-2 virus in clinical set up. Hence, they need to keep themselves updated with any new information regarding this disease.

2. Dental settings should balance the need to provide necessary services while minimizing risk to patients and dental healthcare personnel (DHCP) with recommended measures like regular upgradation of their knowledge and skills regarding infection prevention and control, implementation of triage protocols, encourage physical distancing, PPE supply 
optimization etc. For a detailed guideline for dental practice see Guidelines for Dental professionals in COVID-19 by Ministry of Health and Family Welfare, India ${ }^{1}$ and Guidance for dental settings by Centres for Disease Control and Prevention. 2

3. Until the COVID-19 vaccine is available, personal protective equipment (PPE) and infection prevention and control strategies will play a key role in prevention of transmission or cross-infection with (SARS)-CoV- 2 virus. A buddy system which involves a team of two or more persons who share responsibilities for his/her partner's safety and well-being in the context of (i) appropriately donning and doffing of PPEs, (ii) maintaining hand hygiene and (iii) taking requisite steps on observing breach of PPEs can be followed to ensure prevention of breach of infection prevention and control. In clinical set up the dentist and the auxiliary staff can act as buddy to ensure there is no breach in infection prevention control practices. ${ }^{1}$

4. Usage of PPEs, sanitization and disinfection at regular intervals, single use chair covers increases the cost of providing dental care to the patient, the increased cost can be shared by the patient and the dentist. Also, the government can provide fiscal support by providing subsidised quality PPEs,

5. Throughout this crisis the dental community has continued to work together in partnership with dental council of India and Indian dental Association to ensure the best quality education and treatment are available to dental students and patients respectively. It is also required that all the state and national level representative dental bodies keep work in tandem along with the government to mitigate the impact of COVID- 19.

6. Restructuring of dental education is needed to equip the dental graduates with technological and scientific advancements. Dental graduates should be skilled enough to diagnose basic health conditions or read ECG/CT scan, the focus should be laid more on teaching of evidencebased medicine and dentistry to enable them to better understand the co-dependence of dental health and overall health, thus, preparing them better in dealing with any medical emergencies in their practice and they can also be utilized in case of national health emergencies like COVID-19 to provide basic health care services.

7. Usage of tele-dentistry which is gaining a stronger foothold and changing the outlook of dentistry. Teledentistry can be incorporated as an adjunct into routine dental practice as it offers a wide range of applications like tele-triaging, teleconsultation, tele- diagnosis, telemonitoring etc.

8. Technological innovations in clinical practice. As we move towards resumption of routine dental care, we need to find creative ways to provide safe oral health care for patients.

There is uncertainty surrounding the timeline for the resolution of this pandemic and this health emergency will not be the last one the dental fraternity will be facing, thus we must be better equipped for any such health emergency in the future

\section{References}

1. Ministry of Health and Family Welfare, India. Guidelines for Dental Professionals in COVID-19 situation; 2020. Available from: https:// www.mohfw.gov.in/pdf/DentalAdvisoryF.pdf.

2. Centres for Disease Control and Prevention. Guidance for Dental Settings; 2020. Available from: https://www.cdc.gov/coronavirus/ 2019-ncov/hcp/dental-settings.html.

Cite this article: Sachdev V. COVID-19: Challenges, opportunities, and way ahead for dentistry. J Dent Spec 2020;8(1):1-2. 